\title{
The Ultra-High-Frequency QRS Dyssynchrony in the Assessment of Cardiac Resynchronization Therapy Effect
}

\author{
Pavel Leinveber ${ }^{1}$, Josef Halamek ${ }^{2}$, Pavel Jurak ${ }^{2}$, Magdalena Matejkova ${ }^{1}$, Filip Plesinger ${ }^{2}$, Radovan \\ Smisek $^{2}$, Jolana Lipoldova ${ }^{1}$, Miroslav Novak ${ }^{1}$ \\ ${ }^{1}$ International Clinical Research Center, 1st Cardio-Angiology Department, St Anne’s University \\ Hospital, Brno, Czech Republic \\ ${ }^{2}$ Institute of Scientific Instruments, CAS, Brno, Czech Republic
}

\begin{abstract}
Objective assessment of the effect of Cardiac Resynchronization Therapy (CRT) still represents a very difficult clinical task. The ultra-high-frequency electrocardiographic (ECG) dyssynchrony (e-DYS) represents a simple ECG derived method that provides information about the ventricular electrical depolarization and thus could become helpful in CRT. The 12-lead ECG (sampling $5 \mathrm{kHz} / 24$ bits) and echocardiography were obtained in 42 consecutive CRT patients. The e-DYS and standard QRS duration (QRSd) were automatically assessed before, just after, and 6 months after the CRT during CRT Off and CRT On. In 30 CRT responders determined by echocardiography, the e-DYS parameter was significantly lower after 6 months during CRT Off compared to state prior CRT $(-9 \pm 19 m s, p<0.05)$, while there was no significant e-DYS change during CRT On within 6 months $(-1 \pm 11 \mathrm{~ms}$, NS). A clear shortage of $e$ DYS was observed just after CRT On $\left(-46 \pm 23 m s, p<10 e^{-}\right.$ $\left.{ }^{10}\right)$, and 6 months after CRT On $\left(-46 \pm 21 \mathrm{~ms}, p<10 e^{-10}\right)$ compared to state before CRT. No significant changes of the e-DYS parameter were found in 12 CRT nonresponders. The QRSd didn't show any significance in all observed changes. The e-DYS parameter seems to be a superior parameter to simply and quickly assess an immediate as well as the long-term effect in CRT.
\end{abstract}

\section{Introduction}

It is well known that CRT improves cardiac function and decreases mortality in heart failure patients mainly with wide QRS complex and LBBB morphology [1]. The positive effect of CRT can be evaluated in many ways, from the improvement of cardiac function and symptoms to the reduction of morbidity and mortality. But the CRT effect is primarily induced by electrical stimulation, which is the activation point for the mechanical contraction.
Imaging methods can evaluate the motion of the muscle, but do not detect the underlying mechanisms of dyssynchronous contraction. Ventricular dyssynchrony induced by electrical dyssynchronous depolarization of ventricles can be targeted exactly by CRT [2]. Precise determination of the electrical depolarization of ventricles thus seems to be an important factor for selecting the patients positively responding to CRT.

It has been previously demonstrated that the ECG signal also contains higher frequency components in the QRS complex [3]. The temporal and spatial distribution of these ultra-high-frequency QRS (UHF-QRS) components (from $150 \mathrm{~Hz}$ to $1 \mathrm{kHz}$ ) can describe a ventricular electrical depolarization using only standard ECG 12-lead setup [4]. UHF-QRS electrical dyssynchrony parameter (e-DYS) that describes any delayed electrical activation in segments located below the precordial ECG electrodes can be computed from the temporal distribution of the QRS components.

Since vast majority of published literature presents that CRT effect significantly depends on the specific pattern of ventricular depolarization (the main benefit comes from complete left bundle branch block - LBBB), this study aimed to test the ability of the e-DYS parameter as a simply derived parameter to assess the short and longterm CRT effect against standardly used QRS duration (QRSd).

\section{Methods}

The 12-lead ECG with sampling frequency $5 \mathrm{kHz}$ and amplitude resolution 24bits (SciSDA14, M\&I s r.o., Prague, Czech Republic) was measured in 42 patients ( 8 women) consecutively treated by CRT. The precordial V1-V6 QRS envelopes were calculated through various frequency ranges from 150 to $1000 \mathrm{~Hz}$ using signal averaging technique to improve the signal-to-noise ratio, and electrical depolarization maps were created as described in [4]. The e-DYS was automatically measured 
as a difference between the soonest and latest activation in V leads. The standard QRS duration (QRSd) was also automatically assessed using the custom-made software [5]. All the parameters were assessed before (Before CRT), just after (CRT Off and On), and 6 months after the CRT (6M CRT Off and On).

Echocardiography (Vivid E9, GE Healthcare, Wauwatosa, WI) was also performed in all patients, and those with a relative decrease of $10 \%$ and more in the end-systolic volume of the left ventricle after 6 month period were considered CRT responders.

\section{Results}

From all 42 CRT patients, 30 were determined as positive responders to the therapy by the echocardiographic results.

Mean values (Table 1.) of e-DYS and QRSd parameters were significantly lower in non-responders before CRT as well as just after CRT during CRT Off. eDYS parameter was also significantly lower in CRT nonresponders 6 months after CRT (6M CRT Off).

Table 1. Mean values [ms] of QRS duration (QRSd) and electrical ventricular dyssynchrony (e-DYS) parameters before (Before CRT), just after (CRT Off and On), and 6 months after the CRT (6M CRT Off and On) divided into CRT responders $(\mathrm{R})$ and non-responders (NR). The values are represented as a mean $\pm \mathrm{SD}$.

\begin{tabular}{llrrc}
\hline \multicolumn{2}{l}{ Mean values [ms] } & \multicolumn{1}{c}{ R } & \multicolumn{1}{c}{ NR } & p \\
\hline \multirow{2}{*}{ Before CRT } & QRSd & $167 \pm 16$ & $153 \pm 19$ & $<\mathbf{0 . 0 5}$ \\
& e-DYS & $71 \pm 21$ & $36 \pm 22$ & $<\mathbf{0 . 0 0 0 1}$ \\
CRT Off & QRSd & $167 \pm 18$ & $154 \pm 17$ & $<\mathbf{0 . 0 5}$ \\
\multirow{4}{*}{ CRT On } & e-DYS & $71 \pm 22$ & $35 \pm 21$ & $<\mathbf{0 . 0 0 0 1}$ \\
\multirow{5}{*}{ 6M CRT Off } & QRSd & $158 \pm 29$ & $157 \pm 25$ & NS \\
\multirow{4}{*}{ 6M CRT On } & e-DYS & $25 \pm 14$ & $20 \pm 13$ & NS \\
& QRSd & $163 \pm 24$ & $154 \pm 17$ & NS \\
& Q-DYS & $62 \pm 27$ & $36 \pm 23$ & $<\mathbf{0 . 0 1}$ \\
& e-DYS & $157 \pm 25$ & $161 \pm 28$ & NS \\
\hline
\end{tabular}

In 30 CRT responders (Table 2.), the e-DYS parameter was significantly lower after 6 months during CRT Off (6M CRT Off) compared to state prior CRT $(-9 \pm 19 \mathrm{~ms}$, $\mathrm{p}<0.05)$, while there was no significant e-DYS change during CRT On within 6 months $(-1 \pm 11 \mathrm{~ms}$, NS). On the other hand, there was a clear shortage of e-DYS just after CRT On $\left(-46 \pm 23 \mathrm{~ms}, \mathrm{p}<10 \mathrm{e}^{-10}\right)$, and 6 months after CRT On $\left(-46 \pm 21 \mathrm{~ms}, \mathrm{p}<10 \mathrm{e}^{-10}\right)$ compared to state before CRT (Tab. 2). In 12 CRT non-responders, there were no significant changes in the e-DYS parameter with borderline p-value $(0,052)$ in CRT On compared to before CRT (Table 2.).

The QRSd parameter didn't provide any significance in changes before, just after, and 6 months after CRT during CRT On and Off in both CRT groups (Table 3.).
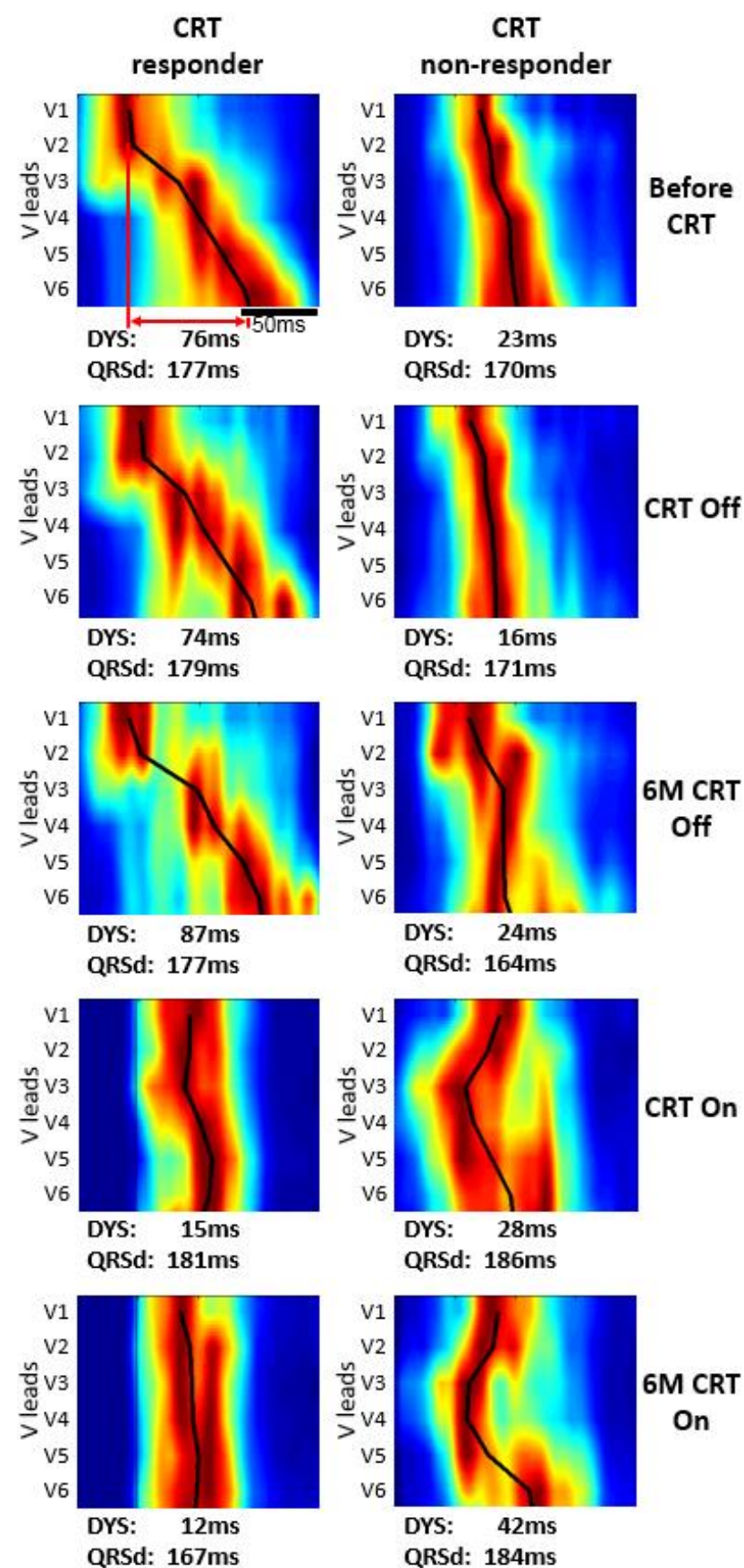

Figure 1. Example of the UHF-QRS electrical depolarization maps from which the e-DYS parameter is automatically calculated in CRT responder and nonresponder. The maps were calculated from the time points before (Before CRT), just after (CRT Off and On), and 6 months after the CRT (6M CRT Off and On). 
Table 2. Changes of the e-DYS parameter in CRT responders (R) and non-responders (NR) in various states before and during CRT. The values are represented as a mean $\pm \mathrm{SD}$.

\begin{tabular}{|c|c|c|}
\hline e-DYS changes [ms] & $\mathbf{R}$ & NR \\
\hline CRT Off - before CRT & $\begin{array}{c}-5 \pm 21 \\
N S\end{array}$ & $\begin{array}{c}-2 \pm 8 \\
\text { NS }\end{array}$ \\
\hline 6M CRT Off - before CRT & $\begin{array}{l}-9 \pm 19 \\
<\mathbf{0 . 0 5}\end{array}$ & $\begin{array}{c}-5 \pm 12 \\
\mathrm{NS}\end{array}$ \\
\hline 6M CRT Off - CRT Off & $\begin{array}{r}-4 \pm 17 \\
<\mathbf{0 . 0 5}\end{array}$ & $\begin{array}{c}-3 \pm 10 \\
\text { NS }\end{array}$ \\
\hline CRT On - before CRT & 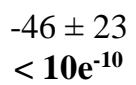 & $\begin{array}{c}-16 \pm 26 \\
N S\end{array}$ \\
\hline 6M CRT On - before CRT & $\begin{array}{r}-46 \pm 21 \\
<\mathbf{1 0} \mathrm{e}^{-10}\end{array}$ & $\begin{array}{c}-12 \pm 25 \\
\mathrm{NS}\end{array}$ \\
\hline 6M CRT On - CRT On & $\begin{array}{c}-1 \pm 11 \\
\mathrm{NS}\end{array}$ & $\begin{array}{c}4 \pm 9 \\
\mathrm{NS}\end{array}$ \\
\hline
\end{tabular}

Table 3. Changes of the QRSd parameter in CRT responders (R) and non-responders (NR) in various states before and during CRT. The values are represented as a mean $\pm \mathrm{SD}$.

\begin{tabular}{lcc}
\hline QRSd changes [ms] & R & NR \\
\hline \multirow{2}{*}{ CRT Off - before CRT } & $0 \pm 8$ & $1 \pm 3$ \\
& NS & NS \\
6M CRT Off - before CRT & $-5 \pm 18$ & $1 \pm 7$ \\
& NS & NS \\
6M CRT Off - CRT Off & $-3 \pm 18$ & $0 \pm 6$ \\
& NS & NS \\
CRT On - before CRT & $-10 \pm 30$ & $5 \pm 28$ \\
\multirow{2}{*}{ 6M CRT On - before CRT } & $-11 \pm 31$ & NS \\
\multirow{2}{*}{ 6M CRT On - CRT On } & $-1 \pm 24$ & NS \\
& NS & NS \\
\hline
\end{tabular}

\section{Discussion}

Objective assessment of the effect of CRT is a very difficult clinical task that still has not been fully clarified. CRT influences cardiac function through the electrical stimulation of the myocardium. But strong parameters describing the electrical properties convenient for CRT are still missing.

QRSd represents basic and clinically simple parameter that can evaluate some electrical conduction disturbances in ventricles. But QRSd evinces a rather poor accuracy in detecting an interventricular and intraventricular dyssynchrony [6].

Various definitions for LBBB can be used in clinical practice. But the response to the CRT is influenced by the criteria for the LBBB definition [7]. Also, only $70 \%$ of LBBB morphology diagnosed patients have echocardiographic evidence of mechanical dyssynchrony [6]. Vectorcardiographic QRS area represents another currently promising method reflecting a long-term CRT outcome [8,9]. But all these parameters are only surrogates for the assessment of electrical ventricular dyssynchrony.

UHF-QRS electrical dyssynchrony parameter (e-DYS) is also derived from standard 12-lead ECG setup but measures the differences of the electrical ventricular depolarization directly in milliseconds.

In our results, the mean values of both tested parameters - e-DYS and QRSd are very consistent before and just after the pacemaker implantation during CRT Off. QRSd is significantly higher in responders, which corresponds with general clinical knowledge. Similarly, e-DYS is also significantly higher in responders confirming our previous findings that patients with higher ventricular dyssynchrony more benefit from the therapy [10]. But the e-DYS shows much higher differences between CRT responders and non-responders. This fact favors the e-DYS parameter as a CRT response predictor against the QRSd parameter.

The significantly lower e-DYS after 6 months followup during switched-off stimulation (6M CRT Off - before CRT) in CRT responders shows the positive change in native electrical ventricular conduction and could be a marker of reverse electrical remodeling [11].

When comparing the e-DYS parameter between stimulation and state prior CRT (CRT On - before CRT), there is a very clear shortage of the dyssynchrony in CRT responders caused by the proper function of the pacemaker as an electrical re-synchronization of the ventricles.

But interestingly no significant change occurs when comparing e-DYS within the follow-up during pacing (6M CRT On - CRT On). Stable and unchanged pacing pattern of an electrical depolarization in time means that the effect of the CRT can be tested just after the pacemaker implantation or even better still during the implantation.

QRSd parameter did not show any significant differences in both CRT responders as well as nonresponders. There was only a trend $(p=0.065)$ towards a significant difference in paced QRSd after 6 months compared to the state before CRT (6M CRT On - before CRT) in the CRT responders group. The results published in similarly oriented studies report significant changes in QRSd before and after CRT [12,13]. The discrepancy in QRSd behavior during CRT with other published data can be caused by the method of QRSd assessment (fully automatic analysis in our case vs. manually assessed QRSd in other articles). 


\section{Conclusion}

The future CRT benefit can be possibly estimated by the value of the e-DYS parameter before CRT, and also immediately after the pacemaker implantation because the electrical ventricular conduction, caused by stimulation, does not substantially change with time. Some kind of reverse electrical remodeling could be evaluated from the comparison between e-DYS values during CRT Off over time.

The ultra-high-frequency QRS dyssynchrony parameter accurately describes the ventricular conduction pattern and seems to be a superior parameter to simply and quickly assess an immediate as well as long-term (reverse electrical remodeling) effect in CRT. e-DYS is the only parameter that simply and noninvasively measures ventricular electrical dyssynchrony. The QRS duration as a descriptor of the ventricular conduction changes induced by CRT does not provide sufficiently valuable information.

\section{Acknowledgments}

The research was supported by Czech Science Foundation, project GA17-13830S, by Ministry of Education, Youth and Sports of the Czech Republic (project LO1212 and LQ1605) and by the Czech Academy of Sciences (project RVO:68081731).

\section{References}

[1] Brignole M, Auricchio A, Baron-Esquivias G, Bordachar P, Boriani G, et al. 2013 ESC Guidelines on cardiac pacing and cardiac resynchronization therapy: the Task Force on cardiac pacing and resynchronization therapy of the European Society of Cardiology (ESC). Developed in collaboration with the European Heart Rhythm Association (EHRA). Eur Heart J 2013;34:2281-2329.

[2] Abraham T, Kass D, Tonti G, Tomassoni GF, Abraham WT, et al. Imaging cardiac resynchronization therapy. JACC Cardiovasc Imaging 2009;2:486-497.

[3] Jurak P, Halamek J, Leinveber P, Vondra V, Soukup L, et al. Ultra-high-frequency ECG measurement. 2013 Computing in Cardiology Conference 2013;40:783-786.

[4] Jurak P, Halamek J, Meluzin J, Plesinger F, Postranecka $\mathrm{T}$, et al. Ventricular dyssynchrony assessment using ultrahigh frequency ECG technique. Journal of Interventional Cardiac Electrophysiology 2017;49:245-254.

[5] Vitek M, Hrubes J, Kozumplik J. A wavelet-based ECG delineation with improved $\mathrm{P}$ wave offset detection accuracy. Analysis of Biomedical Signals and Images 2010;160-165.

[6] Bleeker GB, Schalij MJ, Molhoek SG, Verwey HF, Holman ER, et al. Relationship between QRS duration and left ventricular dyssynchrony in patients with end- stage heart failure. Journal of Cardiovascular Electrophysiology 2004;15:544-549.

[7] Caputo ML, van Stipdonk A, Illner A, D'Ambrosio G, Regoli F, et al. The definition of left bundle branch block influences the response to cardiac resynchronization therapy. International Journal of Cardiology 2018;269:165-169.

[8] Emerek K, Friedman DJ, Sorensen PL, Hansen SM, Larsen JM, et al. Vectorcardiographic QRS area is associated with long-term outcome after cardiac resynchronization therapy. Heart Rhythm 2019;16:213219.

[9] Halamek J, Leinveber P, Viscor I, Smisek R, Plesinger F, et al. The relationship between ECG predictors of cardiac resynchronization therapy benefit. Plos One 2019;14.

[10] Plesinger F, Jurak P, Halamek J, Nejedly P, Leinveber P, et al. Ventricular electrical delay measured from body surface ECGs is associated with cardiac resynchronization therapy response in left bundle branch block patients from the MADIT-CRT trial (Multicenter automatic defibrillator implantation-cardiac resynchronization therapy). Circ Arrhythm Electrophysiol 2018;11:e005719.

[11] Leinveber P, Halamek J, Jurak P, Matejkova M, Lipoldova J, et al. Reverse electrical remodeling assessed by high-frequency QRS dyssynchrony and QRS duration. 2018 Computing in Cardiology Conference 2018.

[12] Kamireddy S, Agarwal SK, Adelstein E, Jain S, Saba S. Correlation of electrical and mechanical reverse remodeling after cardiac resynchronization therapy. Ann Noninvasive Electrocardiol 2009;14:153-157.

[13] Molhoek SG, L VANE, Bootsma M, Steendijk P, Van Der Wall EE, et al. QRS duration and shortening to predict clinical response to cardiac resynchronization therapy in patients with end-stage heart failure. Pacing Clin Electrophysiol 2004;27:308-313.

Address for correspondence:

Pavel Leinveber

St. Anne's University Hospital Brno

International Clinical Research Center

Pekařská 53, 65991 Brno, Czech Republic

pavel.leinveber@fnusa.cz 